\title{
Qualidade de vida: comparando resultados em idosos com e sem presbiacusia
}

\author{
Quality of life: comparing results in elderly with and without presbyacusis
}

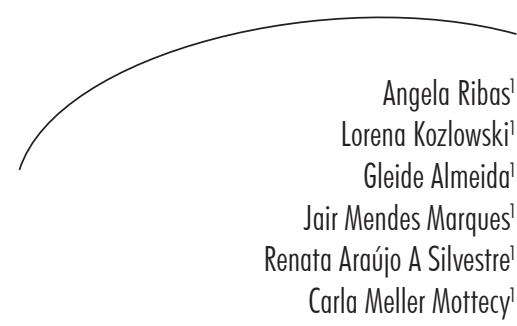

\section{Resumo}

Objetivo: Avaliar a qualidade de vida de um grupo de idosos presbiacúsicos após a protetização e comparar os resultados com um grupo de idosos normouvintes. Método: Estudo clínico descritivo transversal, do qual participaram 51 indivíduos, de ambos os gêneros, divididos em dois grupos: grupo estudo, formado por 36 presbiacúsicos, com idade média de 73 anos, e usuários de prótese auditiva (mínimo de seis meses de uso); grupo controle, formado por 15 idosos normouvintes, com idade média de 69 anos. Foi aplicado o questionário WHOQOL-bref, e os dados relativos aos quatro domínios abrangidos (físico, psicológico, meio ambiente e relações sociais) foram analisados e comparados entre os grupos. Resultados: Qualitativamente, todos os sujeitos presbiacúsicos declararam que sua vida melhorou depois da adaptação da prótese, porém os escores do WHOQOL-breaf revelaram: em relação ao domínio físico, não houve diferença significativa entre as respostas dos dois grupos, certamente porque o avanço da idade traz no seu bojo uma série de limitações; nos demais domínios, houve diferença estatisticamente significante entre os grupos, sendo que no grupo de presbiacúsicos a qualidade de vida foi pior. Tal fato permite inferir que, no grupo de presbiacúsicos, apesar do uso da prótese, as limitações impostas pela idade continuam comprometendo a autoestima, as relações interpessoais e o convívio social. Conclusão: Nos dois grupos, a idade representa limitações no que se refere à qualidade de vida. No grupo com presbiacusia, foi possível verificar melhoria da percepção auditiva, porém a melhora da qualidade de vida geral desses indivíduos depende de outros fatores que podem não ter relação com a perda de audição, quando comparado com o grupo normouvinte.

\section{Abstract}

Objective: To evaluate the quality of life of a group of presbycusic elderly after using the device and comparing the results with a group of normal hearing elderly subjects. Method: This was a cross-sectional clinical study with 51 subjects participants who were divided in two groups: the study group was made up of 36 presbycusic subjects, with an average age of 73 years, of both genders, and hearing aid users (minimum of 6 months of

Palavras-chave: Fonoaudiologia. Perda Auditiva. Idoso. Auxiliares de Audição. Qualidade de Vida.

Programa de Pós-graduação em Distúrbios da Comunicação. Universidade Tuiuti do Paraná. Curitiba, PR, Brasil.

Correspondência / Correspondence

Angela Ribas

E-mail: angela.ribas@utp.br 
use); the control group comprised 15 normal hearing elderly, with average age of 69. We used the WHOQOL-bref, and the data on four areas covered (physical, psychological, environmental and social relations) were analyzed and compared between groups. Results: Qualitatively, all presbycusic subjects stated that their life improved after the use of the prosthesis; however, WHOQOL-bref scores reveal: in relation to the physical domain, there was no significant difference between the responses of the two groups, probably because aging brings with it a number of limitations; in other areas, there was a statistically significant difference between the groups, with the presbycusic group's quality of life being worse. This fact allows us to infer that, in the group of presbycusic subjects, despite the use of the prosthesis, the limitations imposed by age continue to affect self-esteem, interpersonal relationships, and social interaction. Conclusion: In both groups surveyed, age represents limitations regarding quality of life. In the group with presbycusis, it was possible to verify the improvement of auditory perception, but the improvement of overall quality of life of these individuals depends on other factors that may not be related to hearing loss when compared to the normal hearing group.
Key words: Speech. Language and Hearing Sciences. Hearing Loss. Hearing Aids. Elderly. Quality of Life.

\section{INTRODUÇÃO}

O processo do envelhecimento vem sendo objeto de estudo de muitas áreas do conhecimento, haja vista que, em termos mundiais, a tendência do envelhecimento das populações é fato concreto. ${ }^{1,2}$ Profissionais da área da saúde, em especial, se dedicam ao estudo da qualidade de vida desta grande parcela da população mundial, pois o aumento da expectativa de vida traz no seu bojo, em geral, problemas de saúde decorrentes do processo de senescência. ${ }^{3-5}$

A Fonoaudiologia, ciência que estuda a comunicação humana e seus distúrbios, também vem se debruçando sobre estas questões, principalmente no que se refere aos aspectos auditivos, ${ }^{6-8}$ pois na população idosa um sinal característico é a presbiacusia, ou seja, a perda auditiva causada pelo envelhecimento das estruturas auditivas. Em geral, esta entidade clínica afeta os dois ouvidos de forma similar e simétrica, tem origem basococlear e compromete o reconhecimento da fala. Apesar de invisível, essa perda auditiva configura-se como uma doença incapacitante que pode gerar importantes alterações sociais e econômicas. $^{6}$

Este tipo de deficiência auditiva não apenas limita a capacidade de percepção e discriminação dos sons, mas influi diretamente na capacidade de compreensão da linguagem, afetando a capacidade de socialização de uma pessoa e sua família.. Ela pode gerar um transtorno social e psicológico, que influencia as relações interpessoais e de comunicação, privando o indivíduo do convívio com familiares e amigos, levando ao isolamento e comprometendo sua qualidade de vida.

A adaptação de próteses auditivas em indivíduos com perda de audição causada pela presbiacusia tem se mostrado um recurso importante frente à problemática em foco, visto que estas representam uma possibilidade de melhoria da qualidade de vida, fazendo com que esses indivíduos retomem alguns hábitos sociais que se encontravam privados em virtude da diminuição da capacidade de comunicação. No entando, o processo de adaptação à prótese requer tempo e disponibilidade. $\mathrm{O}$ idoso, e muitas vezes seus familiares/cuidadores, deverão aprender a manusear o dispositivo, higienizar, trocar baterias e, principalmente, aprender a ouvir novamente. ${ }^{10}$ Encontros de acompanhamento e aconselhamento serão necessários, com a finalidade de criar familiaridade entre o usuário e o dispositivo, e também com os novos sons, que muitas vezes são diferentes daqueles com que, anteriormente, os ouvidos e cérebro estavam acostumados a lidar. 
Em 2004, preocupado com a saúde auditiva da população brasileira, o governo federal instituiu a Política Nacional de Atenção à Saúde Auditiva (PNASA) considerando, entre outras questões, a necessidade de desenvolver estratégias de promoção da qualidade de vida, educação, proteção e recuperação da saúde e prevenção de danos à audição no Sistema Único de Saúde (SUS). Esta política democratizou o uso da prótese auditiva no Brasil, visto que permitiu às classes socioeconômicas menos favorecidas o acesso a dispositivos eletrônicos considerados caros. ${ }^{11}$

Uma vez que um dos recursos à disposição da população que possui perdas auditivas é a prótese, a PNASA definiu critérios técnicos mínimos para o funcionamento e a avaliação dos serviços que realizam reabilitação auditiva, bem como os mecanismos de sua monitoração com vistas a potencializar os resultados da protetização. A política está presente nos três níveis de atenção à saúde. 1) Atenção Básica: compreende ações de promoção à saúde auditiva, de prevenção e identificação precoce de problemas auditivos junto à comunidade, assim como ações informativas, educativas, orientação familiar e encaminhamentos, quando necessário, para o serviço de atenção à saúde auditiva na média complexidade. 2) Média Complexidade: constitui a primeira referência para a atenção básica e contrarreferência do serviço de atenção à saúde auditiva na alta complexidade. Tem como finalidade prestar assistência especializada às pessoas com doenças otológicas e em especial às pessoas com deficiência auditiva. E 3) Alta Complexidade: constitui referência para o diagnóstico das perdas auditivas e sua reabilitação em crianças até três anos de idade e em pacientes com afecções associadas (neurológicas, psicológicas, síndromes genéticas, cegueira, visão subnormal), perdas unilaterais e daqueles que apresentarem dificuldade na realização da avaliação audiológica em serviço de menor complexidade. Neste sentido, deverá contar com equipamentos para realizar o diagnóstico diferencial das perdas auditivas. ${ }^{11}$
Assim sendo, atualmente é grande o número de usuários idosos do SUS beneficiados com a concessão de próteses auditivas, o que leva os pesquisadores a estudarem e demonstrarem os benefícios auditivos causados pelo uso dos dispositivos. ${ }^{12-14}$

Estudos vêm demonstrando que seu uso favorece a melhora da qualidade de vida em usuários destes aparelhos, ${ }^{7,8,15}$ mas essas pesquisas fazem comparações intragrupo, avaliando as queixas dos respondentes antes e depois da adaptação dos aparelhos. Torna-se, portanto, necessário verificar o impacto da perda auditiva e da protetização em população idosa, em relação a pessoas da mesma faixa etária, porém com audição normal e que, portanto, não usam prótese auditiva.

Queixas de isolamento, depressão e ansiedade, que permeiam a vida de idosos presbiacúsicos, são também comuns àqueles que escutam. Estaria a prótese auditiva, adaptada em indivíduos presbiacúsicos, devolvendo habilidades auditivas importantes a esta população e melhorando, consequentemente, sua qualidade de vida? As queixas associadas à perda auditiva estariam sendo minimizadas pelo uso do dispositivo?

Assim, estabeleceu-se como objetivo deste trabalho avaliar a qualidade de vida de um grupo de idosos presbiacúsicos após a protetização e comparar os resultados com um grupo de idosos normouvintes.

\section{MÉTODO}

Este estudo, do tipo clínico descritivo transversal, foi desenvolvido em Clínica de Fonoaudiologia credenciada pelo SUS como serviço de Alta Complexidade para Saúde Auditiva. Os dados foram coletados no período de junho de 2010 a abril de 2011.

Participaram 51 indivíduos, sendo 37 mulheres e 14 homens, divididos em dois grupos: grupo estudo, formado por 36 presbiacúsicos e usuários 
de prótese auditiva (mínimo de seis meses de uso); e grupo controle, formado por 15 idosos normouvintes. Todos os participantes foram escolhidos aleatoriamente dentre os pacientes da clínica. Para a coleta de dados, aplicou-se o questionário WHOQOL-bref. ${ }^{16}$

A Organização Mundial da Saúde, preocupada em elaborar um protocolo de qualidade de vida aplicável a diferentes populações e culturas, desenvolveu, em 1991, o World Health Organization Quality of Life-100 (WHOQOL-100). Participaram da elaboração deste protocolo 15 centros internacionais. $\mathrm{Na}$ sequência, foi criada a versão abreviada, intitulada WHOQOL-bref, cuja versão final foi composta por 26 questões. ${ }^{16} \mathrm{~A}$ primeira questão refere-se à qualidade de vida de modo geral e a segunda, à satisfação com a própria saúde. As outras 24 estão divididas nos domínios "físico", "psicológico", "relações sociais" e "meio ambiente", sendo um instrumento que pode ser utilizado tanto para populações saudáveis como para aquelas acometidas por agravos e doenças crônicas. Além do caráter transcultural, os instrumentos WHOQOL valorizam a percepção individual da pessoa, podendo avaliar qualidade de vida em diversos grupos e situações.

Para o grupo estudo, foram estabelecidos como critérios de inclusão: ter mais de 60 anos; diagnóstico de presbiacusia; experiência com uso de prótese auditiva de, pelo menos, seis meses; e participar efetivamente das consultas de acompanhamento oferecidas pela clínica. Para o grupo controle, estabeleceram-se como critérios de inclusão: ter mais de 60 anos e não possuir perda auditiva. Foram excluídos indivíduos com perdas auditivas severas e profundas e que não possuíam condições de responder ao questionário.

O questionário WHOQOL-bref foi aplicado ao respondente pela pesquisadora, individualmente, em sala silenciosa, após o atendimento fonoaudiológico. $\mathrm{O}$ profissional leu as questões sem oferecer sinônimos ou interpretações, para não modificar seu sentido original. Caso o respondente tivesse dificuldades para compreender a questão, o profissional repetia a pergunta. Optou-se por esta forma de aplicação, pois a literatura comenta que é comum indivíduos idosos oriundos de classe econômica desfavorecida, apesar de letrados, terem dificuldade em extrair informações de textos simples. ${ }^{17}$ As respostas foram assinaladas no protocolo, que é objetivo, e alguns depoimentos relevantes foram registrados.

Os questionários foram analisados considerando-se os escores gerais (questões $1 \mathrm{e}$ 2) e quatro domínios de abrangência:

1- físico:questões relacionadas a dor, desconforto, fadiga, sono, mobilidade e capacidade para desempenhar tarefas (questões 3, 4, 10, 15, 16, 17, 18);

2- psicológico: questões relacionadas a raciocínio, aprendizagem, concentração, memória, sentimentos e espiritualidade (questões 5, 6, 7, 11, 19, 26);

3- meio ambiente: questões relacionadas com o ambiente do lar e do trabalho, participação, recreação e lazer (questões 8, 9, 12, 13, 14, 23, 24, 25);

4- relação pessoal: questões relativas ao relacionamento interpessoal (questões 20, 21, 22).

Os valores gerais e por domínio foram estabelecidos. Na sequência, compararam-se os achados entre o grupo estudo e o grupo controle. O teste $t$ Student foi aplicado para estabelecer diferenças, sendo considerados significantes os valores inferiores a $0,05 \%$.

O estudo foi aprovado pelo Comitê de Ética em Pesquisa da Universidade Tuiuti do Paraná, sob protocolo no UTP/027/2008. Todos os participantes assinaram o Termo de Consentimento Livre e Esclarecido. 


\section{RESULTADOS}

O grupo estudo contou com 36 participantes, com idade mínima de 61 anos, máxima de 83 e média de 73 anos. Quanto ao gênero, 73\% eram do sexo feminino. No grupo controle, com 15 participantes, a idade mínima foi de 62 anos, a máxima de 79 e a média de 69 anos. Quanto ao gênero, $66 \%$ eram do sexo feminino.

A caracterização dos sujeitos em relação à configuração audiométrica encontra-se na tabela 1, e com relação ao tipo de aparelho adaptado no grupo estudo, encontra-se na tabela 2.

Tabela 1. Caracterização dos grupos em relação à configuração audiométrica ( $n=51)$. Curitiba-PR, 2010-2011.

\begin{tabular}{l|ccc|c|c|}
\hline & \multicolumn{2}{|c|}{ Grupo estudo $(\mathrm{n}=36)$} & \multicolumn{2}{c|}{ Grupo controle $(\mathrm{n}=15)$} \\
\cline { 2 - 5 } & Feminino & Masculino & Feminino & Masculino \\
\hline Limiares normais & 0 & 0 & 10 & 5 \\
Perda leve bilateral & 15 & 2 & 0 & 0 \\
Perda moderada bilateral & 12 & 7 & 0 & 0 \\
\hline Total & 27 & 9 & 10 & 5 \\
\hline
\end{tabular}

Tabela 2. Caracterização do grupo estudo em relação ao uso da prótese auditiva (n=36). Curitiba-PR, 2010-2011.

\begin{tabular}{l|c|c|}
\hline \multirow{2}{*}{$\begin{array}{c}\text { Tecnologia } \\
\text { digital }\end{array}$} & Feminino & Masculino \\
\cline { 2 - 3 } $\begin{array}{l}\text { Adaptação } \\
\text { Binaural }\end{array}$ & 27 & 9 \\
Monoaural & 20 & 5 \\
Modelo & 7 & 4 \\
Intrauricular & & \\
Retroauricular & 15 & 7 \\
\hline
\end{tabular}

Com base na comparação dos resultados do questionário WHOQOL-bref, pode-se notar que a maioria dos pesquisados nos dois grupos referiu, de modo geral, possuir boa qualidade de vida (figura 1).
As respostas para cada questão foram analisadas e os escores obtidos foram comparados entre os dois grupos. Conforme demonstra o figura 2, as médias do grupo controle estão significativamente maiores que no grupo estudo. 


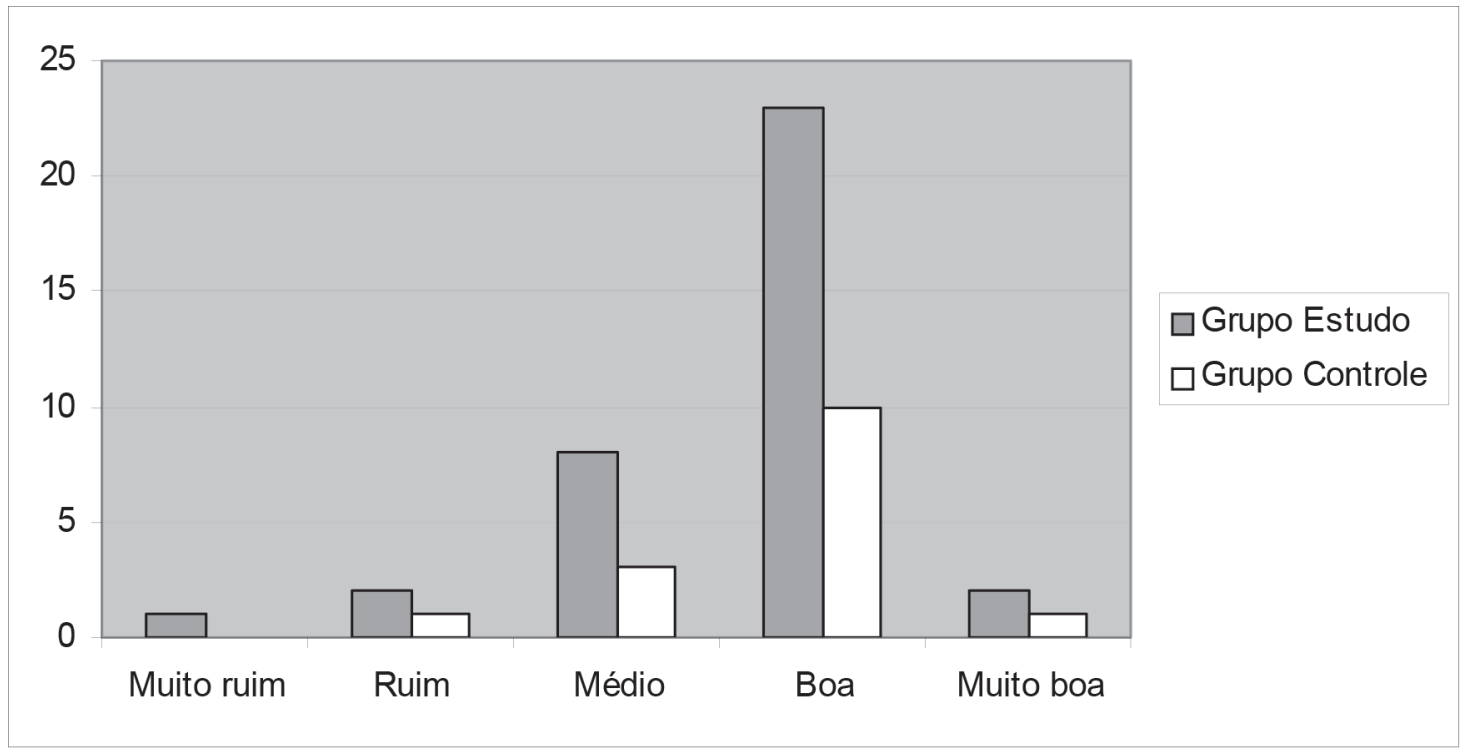

Figura 1. Qualidade de vida geral referida pelos participantes, grupo estudo $(\mathrm{n}=36)$ e grupo controle (n=15). Curitiba-PR, 2010-2011.

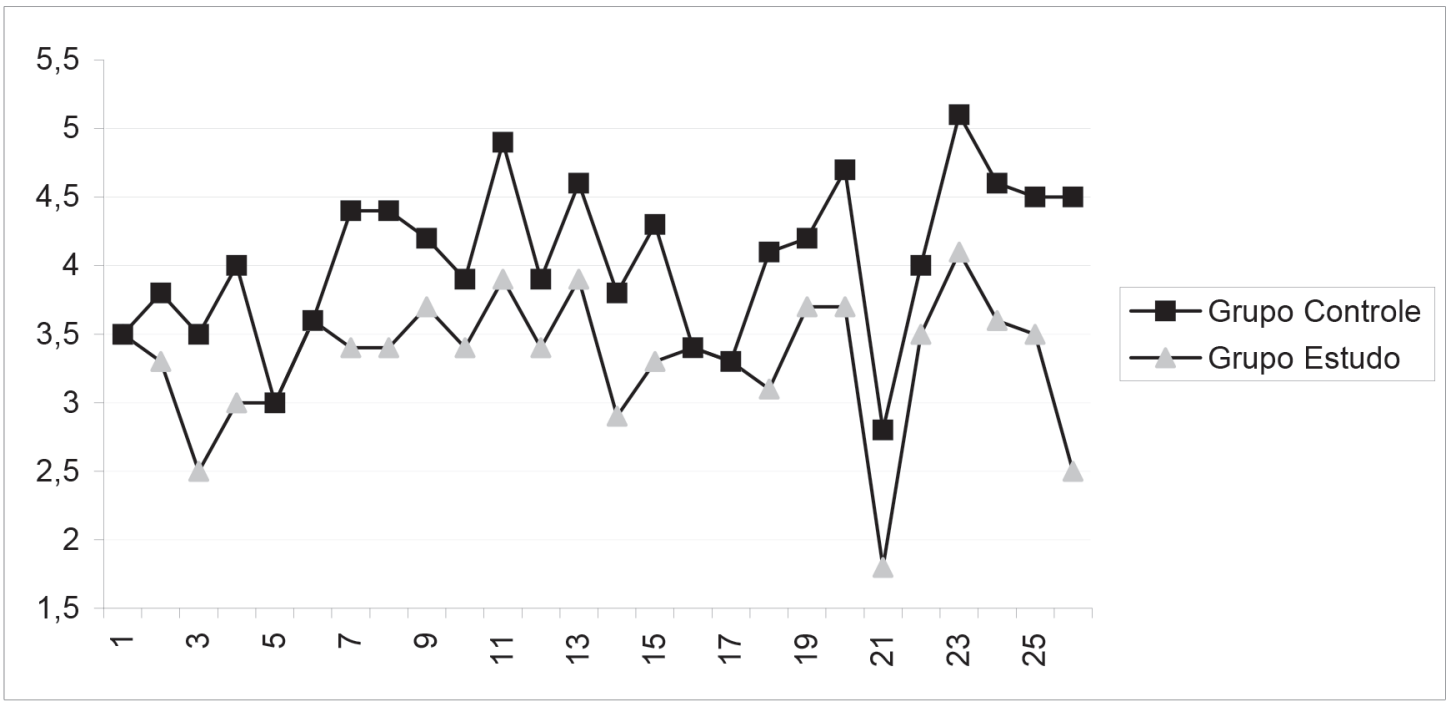

Figura 2. Qualidade de vida geral referida pelos participantes, grupo estudo $(\mathrm{n}=36)$ e grupo controle ( $\mathrm{n}=15)$. Curitiba-PR, 2010-2011.

As respostas por domínio foram analisadas e comparadas entre os grupos. Por meio do teste $t$ Student, ao nível de significância de 0,05 (5\%), verifica-se que existe diferença significativa entre as médias dos escores dos dois grupos para os domínios "meio ambiente" e "relações pessoais", com médias significativamente maiores no grupo controle (tabela 3). 
Tabela 3. Comparação entre os escores médios, por domínio, dos grupos estudo e controle. Curitiba-PR, 2010-2011.

\begin{tabular}{l|ccc|c|c|c}
\hline \multirow{2}{*}{ Domínios } & \multicolumn{2}{|c|}{ Grupo estudo $(\mathrm{n}=36)$} & \multicolumn{2}{c|}{ Grupo controle $(\mathrm{n}=15)$} & \multirow{2}{*}{$\mathrm{p}$} \\
\cline { 2 - 5 } & média & desvio-padrão & média & desvio-padrão & \\
\hline Geral & 3,4 & 0,8 & 3,7 & 0,7 & 0,2114 \\
Físico & 3,2 & 0,5 & 3,1 & 0,5 & 0,4897 \\
Psicológico & 2,5 & 1,1 & 2,8 & 0,7 & 0,3258 \\
Meio ambiente & 2,9 & 0,6 & 3,5 & 0,3 & $0,0012^{*}$ \\
Relações pessoais & 2,8 & 0,7 & 3,3 & 0,4 & $0,0055^{*}$ \\
\hline
\end{tabular}

*Houve diferença significativa.

\section{DISCUSSÃO}

Atualmente, a velhice constitui grande desafio para o homem contemporâneo, pois a sociedade valoriza a produtividade e a força jovial, fazendo muitas vezes com que o idoso se sinta frustrado. Este, durante o processo de envelhecimento, tem que lidar com uma série de desafios, e quando uma perda auditiva impacta sua vida, comunicar-se com os outros se torna um problema que compromete seu relacionamento com familiares e amigos.

O grupo pesquisado neste estudo caracterizou-se por ser presbiacúsico, sendo que houve predominância do gênero feminino na amostra. A literatura consultada revela que as mulheres buscam com maior frequência serviços de saúde auditiva. ${ }^{18}$

Observou-se predominância da perda auditiva de grau moderado bilateral, em que a média das frequências testadas pela audiometria está em torno de 41 a $70 \mathrm{~dB}$. Esta configuração de perda reduz a capacidade de discriminação do som, dificulta a inteligibilidade de fala e, muitas vezes, inviabiliza o uso do telefone. A presença da presbiacusia é comum na faixa etária da amostra estudada, e a perda moderada bilateral é a mais relatada. ${ }^{19}$
Uma das terapêuticas mais indicadas para a reabilitação auditiva é o uso da prótese, estando comprovado que favorece a qualidade de vida geral do indivíduo. ${ }^{8}$ No entanto, a tecnologia, o tipo de adaptação e o modelo do aparelho interferem na qualidade da adaptação.

Neste trabalho, observou-se que todos os participantes do grupo estudo fazem uso da tecnologia digital, a qual proporciona amplificação suave e voltada para as necessidades reais do paciente, visto que os ajustes finos da prótese são realizados por programas de computador bastante sofisticados. ${ }^{10}$ Também foi possível observar que $69 \%$ da amostra possuíam as duas orelhas protetizadas. É consenso que em indivíduos portadores de perda auditiva bilateral, o uso de dois aparelhos é o ideal, pois confere ao usuário a capacidade de localizar eventos sonoros no ambiente e favorece a resolução de frequência. ${ }^{20}$

Quanto ao modelo utilizado, verificou-se que $61 \%$ da amostra utilizavam prótese intracanal, ou seja, a que se adapta dentro do conduto auditivo e favorece questões estéticas. ${ }^{10} \mathrm{Em}$ pesquisas que investigaram o desempenho de próteses auditivas, os piores escores se referem às questões relacionadas à autoimagem e aos estigmas de deficiência auditiva, em que a visibilidade do aparelho auditivo é tida como negativa..$^{21-23}$ 
Com relação à qualidade de vida geral, os dois grupos apresentaram respostas similares; ou seja, consideraram possuir boa qualidade de vida, apesar das limitações impostas pelo processo natural de envelhecimento e da perda auditiva. É importante frisar, no entanto, que a qualidade de vida na terceira idade é um conceito subjetivo e está diretamente relacionado à preservação de relacionamentos e à boa saúde. ${ }^{5}$ Seidl \& Zannon $^{2}$ afirmam que o conceito de qualidade de vida é influenciado pela subjetividade e pela multidimensionalidade, ou seja, são muitos os aspectos a serem considerados pelo respondente.

$O$ que se pode inferir dos dados aqui apresentados é que a população-alvo deste trabalho está ciente das limitações impostas pela velhice e leva isto em consideração ao analisar seu momento vivido, como destacado nas duas citações apresentadas a seguir, extraídas dos depoimentos dos respondentes.

Qualidade de vida? Eu acho que muito boa, afinal eu tenho 74 anos e ainda consigo sair sozinho, faço minhas tarefas em casa, limpo o jardim, cuido de dois netos. É, eu acho que ainda é boa. (PF, 74 anos).

Eu gosto da minha vida. Já não tenho a mesma disposição de antes, quando era jovem, mas dá para fazer muita coisa. Tenho artrose, mas tomo remédio que alivia, e quando não sinto dor faço tudo em casa" (JLL, 67 anos).

Com relação aos quatro domínios investigados pelo WHOQOL-bref, verifica-se que houve diferença significativa entre os grupos para "meio ambiente" e "relações pessoais". Nestes domínios estão envolvidas competências como segurança, proteção, ambiente, convívio, oportunidades de adquirir informações, suporte e apoio social, além de relações pessoais. ${ }^{16} \mathrm{~A}$ diferença observada entre os grupos permite inferir que a perda auditiva, independentemente do uso da prótese, limita o acesso do indivíduo ao meio, impondo as condições restritivas que a dificuldade de comunicação acarreta.

Já para os domínios "físico" e "psicológico", que abrangem questões sobre saúde física em geral e sentimentos negativos, ${ }^{16}$ não houve diferença significativa entre os grupos. A perda auditiva causa depressão e isolamento, porém não é a única causa destes sinais. ${ }^{8}$

Os resultados similares obtidos nos dois grupos, observados no escore geral do instrumento, bem como nos domínios "físico" e "psicológico", permitiram compreender que no grupo estudo a perda auditiva não é percebida como uma doença, um problema de saúde. De acordo com a literatura consultada ${ }^{7-9}$ a perda auditiva característica da presbiacusia não causa dor, desconforto, não interfere no sono, não afeta a memória. Também não é visível, portanto não degenera a autoimagem. Causa, no entanto, problemas de discriminação e compreensão da linguagem, o que afeta as relações interpessoais. $O$ fato de não escutar bem pode contribuir para a instalação de sentimentos de insegurança, porém este sentimento pode estar presente na população idosa em decorrência de outras comorbidades. ${ }^{9,10}$

Desta forma, o uso da prótese auditiva é fundamental para a melhoria da qualidade de vida de indivíduos idosos, mas é importante esclarecer que apesar de usarem seus aparelhos, algumas limitações ainda se impõem, como a dificuldade de compreender a fala em ambiente ruidoso, dificuldades para compreender fala degradada, ou acompanhar uma conversa com mais de dois interlocutores, ${ }^{9}$ por exemplo. Portanto, o acompanhamento e o aconselhamento constantes são essenciais para a superação das dificuldades enfrentadas por esta população.

Sabedores destas questões, os serviços de saúde auditiva, seja da rede privada ou pública, que configuram centros especializados no atendimento ao idoso com perda auditiva, não devem perder de foco que esta população é poliqueixosa e necessita de atendimento multiprofissional. É necessário investir esforços no sentido de valorizar o idoso, ouvir suas demandas e contribuir para a sua efetiva inserção social.

Outros estudos envolvendo a adaptação de próteses auditivas e qualidade de vida na 
população idosa necessitam ser realizados, considerando fatores educacionais, culturais e financeiros, dados não levantados neste trabalho.

\section{CONCLUSÃO}

De modo geral, a pesquisa demonstrou que, na amostra estudada, os idosos com ou sem perda auditiva acreditam ter qualidade de vida boa, certamente porque o grupo pesquisado possui consciência das dificuldades inerentes ao processo de envelhecimento.

O grupo com perda auditiva, apesar de utilizar a prótese, demonstrou ter piores resultados no

\section{REFERÊNCIAS}

1. Frumi C, Celik KLS. O olhar do idoso frente ao envelhecimento e a morte. Rev Bras Cien Envelhecimento Humano 2006; 23(1):92-100.

2. Seidl EMF, Zannon CMLC. Qualidade de vida e saúde: aspectos conceituais e metodológicos. Cad Saúde Pública 2004; 20(2):580-8.

3. Souza LM, Lautert L, Hilleshein EF. Qualidade de vida e trabalho voluntário em idosos. Rev da Escola de Enfermagem da USP 2010; 16(1):11-17.

4. Campos MO, Neto JFR. Qualidade de vida: um instrumento para promoção de saúde. Rev Baiana de Saúde Pública 2008; 32(2):232-40.

5. Vecchia RD et al. Qualidade de vida na terceira idade: um conceito subjetivo. Rev Bras Epidemiol 2005;8(3):246-52.

6. Teixeira AR et al. Qualidade de vida de adultos e isosos pós adaptação de próteses auditivas. Rev Soc Bras Fonoaudiol 2008;13(4):357-61.

7. Silman $\mathrm{S}$ et al. Próteses auditivas: um estudo sobre seu benefício na qualidade de vida de indivíduos portadores de perda auditiva neurossensorial. Rev Dist Com 2004;16(2):153-65.

8. Mondelli MFCG, Souza PJS. Qualidade de vida antes e após a adaptação do AASI. Braz J Otorhinolaryngol 2012;78(3):1-9.

9. Russo ICP. Intervenção Fonoaudiológica na Terceira Idade. Rio de Janeiro: Revinter; 2004. que se refere aos domínios "meio ambiente" e "relação social", do que o grupo com audição normal, o que permite inferir que a perda auditiva continua impondo sérias limitações ao indivíduo, limitações muitas vezes não amainadas pelo uso da prótese.

Tal fato permite inferir que os profissionais da área da Geriatria e Gerontologia devem enxergar a perda auditiva de seus clientes e buscar recursos para minimizar suas consequências, sem contudo concluir que a protetização dará conta da resolução de todos os problemas associados à dificuldade de ouvir. O acompanhamento e o aconselhamento constantes são essenciais para a superação das dificuldades enfrentadas por essa população.

10. Almeida K, Iorio MCM. Próteses auditivas: fundamentos teóricos e aplicações clinicas. São Paulo: Lovise; 2003.

11. Brasil. Portaria $M S$ no 587 - Determina que as Secretarias de Estado da Saúde dos estados adotem as providências necessárias à organização e implantação das Redes Estaduais de Atenção à Saúde Auditiva. Brasilia: Ministério da Saúde, 2004.

12. Lanzarini I et al. A percepção dos familiares de usuários de próteses auditivas em relação à perda auditiva e suas limitações.Tuiuti Ciên Cult 2013;46(1):117-27.

13. Fialho IM et al. Percepção de idosos sobre uso de AASI concedido pelo SUS. Reev CEFAC 2009;11(2)20-31.

14. Buriti AKL, Oliveira SHS. Adaptação à prótese auditiva em usuários assistidos pelo SUS. Rev Soc Bras Fonoaudiol 2012;17(1):41-6.

15. Teixeira CF, Augusto LGS, Neto SSC. Prótese auditiva: satisfação do usuário com sua prótese e com o meio ambiente. Rev CEFAC 2008;10(2):245-53.

16. Fleck MPA et al. Aplicação da versão em português do instrumento abreviado de qualidade de vida WHOQOL-Bref. Rev Saúde Públ 2000;34(2)178-83.

17. Massi GA et al. Práticas de letramento no processo de envelhecimento. Reev Bras Geriatr Gerontol 2010;13(1):59-72. 
18. Ribas A et al. Perfil sociohistórico de pacientes atendidos na clínica de fonoaudiologia da UTP. Rev Biociências, Tecnologia e Saúde 2012;5(1):13-22.

19. Ruschel CV, Carvalho CR, Guarinello AC. A eficiência de um programa de reabilitação audiológica em idosos com presbiacusia e seus familiares. Rev Soc Bras Fonoaudiol 2007;12(2):95-8.

20. Russo IC, Almeida K. Considerações sobre a seleção e adaptação de próteses auditivas para idoso. In: Almeida K, Iório MCM. Próteses auditivas: fundamentos e aplicações clínicas. São Paulo: Lovise; 1996.
21. Carvalho JSA. Satisfação de Idosos com Aparelhos Auditivos Concedidos no Estado do Tocantins. Intl Arch Otorhinolarygol. 2007;11(4):416-26.

22. Santos JMS, Cabral TP. Análise de Satisfação dos usuários de Próteses Auditivas em seu dia-a-dia. Acta Otorrinolaringol. 2007;25(1):34-7.

23. Farias RB, Russo IPC. Saúde Auditiva: Estudo do Grau de Satisfação de Usuários de Aparelho de Amplificação Sonora Individual. Rev Soc Bras Fonoaudiol. 2010; 15(1): 26-31. 